\title{
Adding a third component with reduced miscibility and higher LUMO level enables efficient ternary organic solar cells
}

Ruijie Ma, ${ }^{\# \mathrm{a}, \mathrm{b}}$ Tao Liu, ${ }^{* \# \mathrm{a}, \mathrm{b}}$ Zhenghui Luo, ${ }^{\mathrm{a}, \mathrm{b}, \mathrm{i}}$ Ke Gao, ${ }^{* \mathrm{c}, \mathrm{f}}$ Kai Chen, ${ }^{\mathrm{d}}$ Guangye

Zhang, ${ }^{* \mathrm{e}}$ Wei Gao, ${ }^{\mathrm{f}, \mathrm{i}}$ Yiqun Xiao, ${ }^{\mathrm{g}}$ Tsz-Ki Lau, ${ }^{\mathrm{g}}$ Qunping Fan, ${ }^{\mathrm{h}}$ Yuzhong Chen, ${ }^{\mathrm{a}, \mathrm{b}}$ Lik-

Kuen Ma, ${ }^{\mathrm{a}, \mathrm{b}}$ Huiliang Sun, ${ }^{\mathrm{a}}$ Guilong Cai, ${ }^{\mathrm{g}}$ Tao Yang, ${ }^{\mathrm{j}}$ Xinhui Lu, ${ }^{\mathrm{g}}$ Ergang Wang, ${ }^{\mathrm{h}}$

Chuluo Yang, ${ }^{\mathrm{i}}$ Alex K-Y. Jen, ${ }^{\mathrm{c}, \mathrm{f}}$ He Yan*a,b

aDepartment of Chemistry and Hong Kong Branch of Chinese National Engineering Research Center for Tissue Restoration \& Reconstruction, Hong Kong University of Science and Technology (HKUST), Clear Water Bay, Kowloon, Hong Kong, China.

${ }^{b}$ Hong Kong University of Science and Technology-Shenzhen Research Institute, No. 9 Yuexing first RD, Hi-tech Park, Nanshan, Shenzhen 518057, P. R. China

${ }^{\mathrm{c}}$ Department of Materials Science and Engineering University of Washington Seattle, WA 98195-2120, USA.

${ }^{\mathrm{d} C o l l e g e}$ of Pharmacy, Xi'an Jiaotong University, No.76 Yanta West Road, Xi'an, Shaanxi Province 710061, People's Republic of China.

efFlexPV Limited, Flat/RM B, 12/F, Hang Seng Causeway Bay BLDG, 28 Yee Wo Street, Causeway Bay, Hong Kong, China.

fDepartment of Chemistry, City University of Hong Kong, Kowloon, Hong Kong

gDepartment of Physics, Chinese University of Hong Kong, New Territories, Hong Kong, China.

hDepartment of Chemistry and Chemical Engineering, Chalmers University of Technology, Göteborg SE-412 96, Sweden

iDepartment of Chemistry Hubei Key Lab on Organic and Polymeric Optoelectronic Materials, Wuhan University, Wuhan 430072, P. R. China

jCentre for Mechanical Technology and Automation, Department of Mechanical Engineering, University of Aveiro, 3810-193 Aveiro, Portugal 


\section{Characterization}

UV-vis absorption spectra were measured using a Shimadzu UV-2500 recording spectrophotometer. Cyclic voltammetry (CV) measurements of targeted SMA thin films were conducted on a CHI voltammetric analyzer in acetonitrile solution with 0.1 M tetrabutylammonium hexafluorophosphate (n-Bu4NPF6) as supporting electrolyte at room temperature by using a scan rate of $100 \mathrm{mV} \mathrm{s}-1$ and conventional three-electrode configuration. AFM measurements were obtained by using a Dimension Icon AFM (Bruker) in a tapping mode. The grazing incidence X-ray scattering (GIWAXS) measurement was carried out with a Xeuss 2.0 SAXS/WAXS laboratory beamline using a Cu X-ray source $(8.05 \mathrm{keV}, 1.54 \AA)$ and a Pilatus3R 300K detector. The incident angle was $0.2^{\circ}$. The samples for GIWAXS/GISAXS measurements were fabricated on silicon substrates using the same recipe for the devices.

\section{Solar cell fabrication and characterization}

Solar cells were fabricated in a conventional device configuration of ITO/PEDOT:PSS/active layers/PNDIT-F3N/Ag. ${ }^{[1]}$ The ITO substrates were first scrubbed by detergent and then sonicated with deionized water, acetone and isopropanol subsequently, and dried overnight in an oven. The glass substrates were treated by UV-Ozone for 30 min before use. PEDOT:PSS (Heraeus Clevios P VP AI 4083) was spin-cast onto the ITO substrates at $4000 \mathrm{rpm}$ for $30 \mathrm{~s}$, and then dried at 150 ${ }^{\circ} \mathrm{C}$ for 15 min in air. The PM6: acceptor blends (1:1.2 weight ratio) were dissolved in chloroform (the total concentration of blend solutions was $14 \mathrm{mg} \mathrm{mL}^{-1}$ for all blends), with 1-chloronaphthalene $(0.5 \% \mathrm{vol})$ as additive, and stirred overnight in a nitrogenfilled glove box. The $55^{\circ} \mathrm{C}$ blend solution was spin-cast at $3000 \mathrm{rpm}$ for $30 \mathrm{~s}$ onto PEDOT:PSS films followed by a temperature anealing of $90^{\circ} \mathrm{C}$ for $5 \mathrm{~min}$. A thin PNDIT-F3N layer was coated on the active layer, followed by the deposition of $\mathrm{Ag}$ $(100 \mathrm{~nm})$ (evaporated under $5 \times 10^{-5} \mathrm{~Pa}$ through a shadow mask). The optimal active layer thickness measured by a Bruker Dektak XT stylus profilometer was about 100 $\mathrm{nm}$. The current density-voltage $(J-V)$ curves of all encapsulated devices were measured using a Keithley 2400 Source Meter in air under AM 1.5G $\left(100 \mathrm{~mW} \mathrm{~cm}^{-2}\right)$ using a 
Newport solar simulator. The light intensity was calibrated using a standard Si diode (with KG5 filter, purchased from PV Measurement to bring spectral mismatch to unity). Optical microscope (Olympus BX51) was used to define the device area $\left(4.6 \mathrm{~mm}^{2}\right)$. EQEs were measured using an Enlitech QE-S EQE system equipped with a standard Si diode. Monochromatic light was generated from a Newport 300W lamp source.

\section{SCLC Measurements}

The electron and hole mobility were measured by using the method of space-charge limited current (SCLC) for electron-only devices with the structure of ITO/ZnO/active layer/PNDIT-F3N/Ag and hole-only devices with the structure of ITO/MoO $/$ active layers $/ \mathrm{MoO}_{\mathrm{x}} / \mathrm{Ag}$. The charge carrier mobility was determined by fitting the dark current to the model of a single carrier SCLC according to the equation: $J=9 \varepsilon_{0} \varepsilon_{\mathrm{r}} \mu V^{2} / 8 d^{3}$, where $J$ is the current density, $d$ is the film thickness of the active layer, $\mu$ is the charge carrier mobility, $\varepsilon_{\mathrm{r}}$ is the relative dielectric constant of the transport medium, and $\varepsilon_{0}$ is the permittivity of free space. $V=V_{\text {app }}-V_{\mathrm{bi}}$, where $V_{\mathrm{app}}$ is the applied voltage, $V_{\mathrm{bi}}$ is the offset voltage. The carrier mobility can be calculated from the slope of the $J^{1 / 2} \sim V$ curves.

\section{Surface Tension Characterization}

Contact angle measurements were carried out by an Attension Theta Flex meter, using water and ethylene glycol by sessile drop analysis. The surface tension values of films are calculated through Fowkes approximation, ${ }^{[2]}$ in which:

$(\cos \theta+1)^{2}=4 \gamma_{S}{ }^{d} \gamma_{L}{ }^{d} / \gamma_{L}{ }^{2}$, where $\gamma_{S}{ }^{d}$ and $\gamma_{L}{ }^{d}$ refer to dispersion tension of plane surface and liquid, while $\gamma_{\mathrm{L}}$ refers to total tension of liquid. 

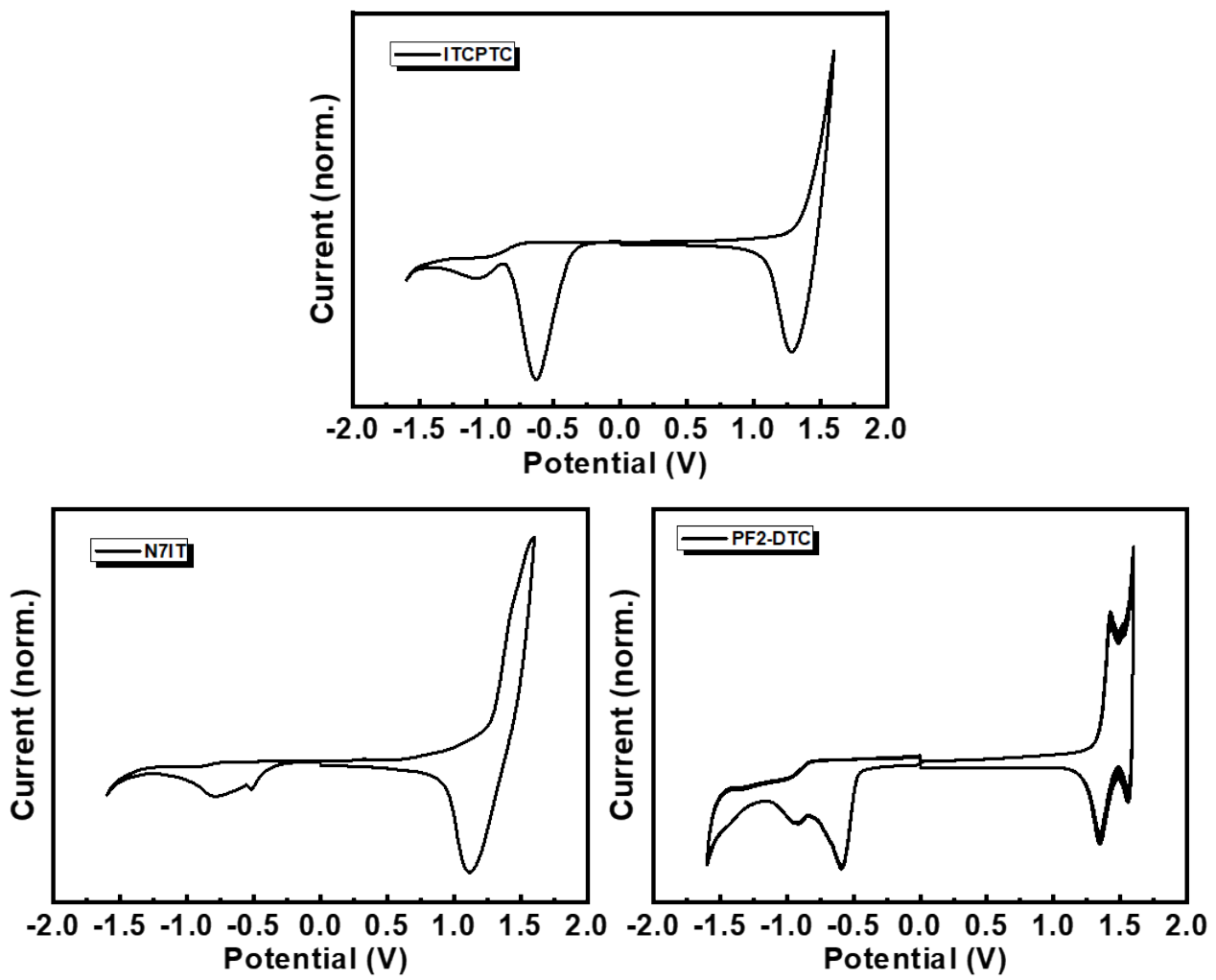

Figure S1. CV curves of ITCPTC, N7IT and PF2-DTC.
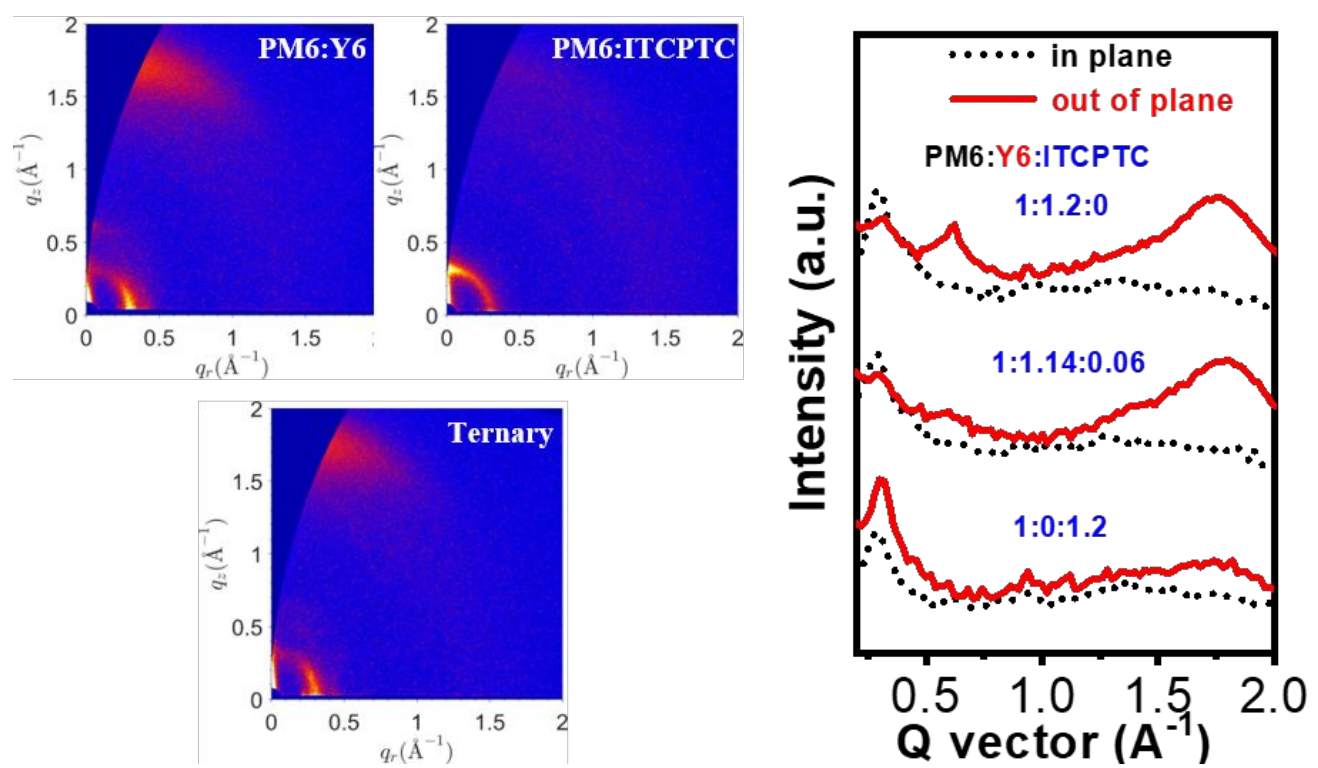

Figure S2. 2D-GIWAXS patterns and corresponding intensity profiles. 
(a)

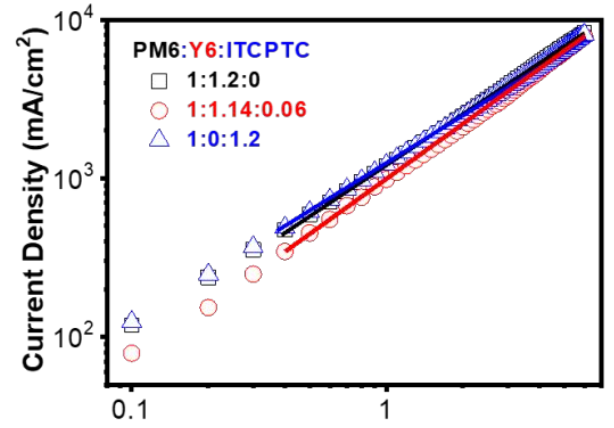

(c)

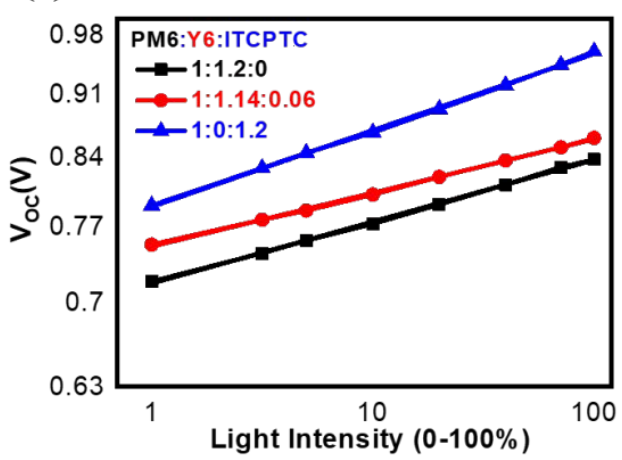

(b)

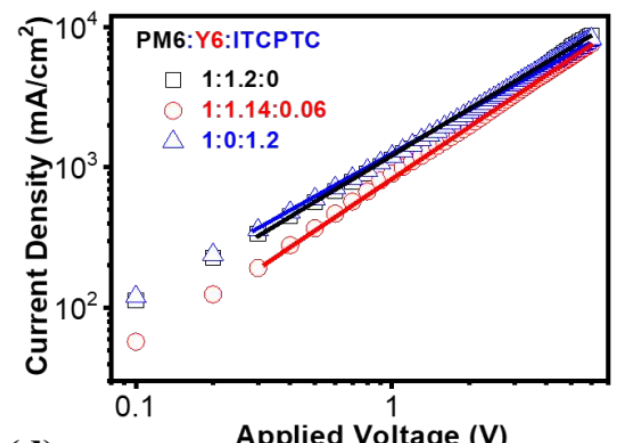

(d)

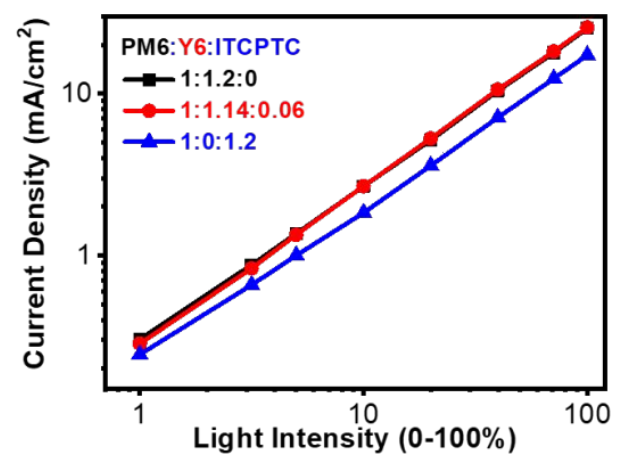

Figure S3. (a) Hole only and (b) electron only device results. (c) $V_{\mathrm{OC}}$ and (d) $J_{\mathrm{SC}}$ versus light intensity relationship curves.

(a)

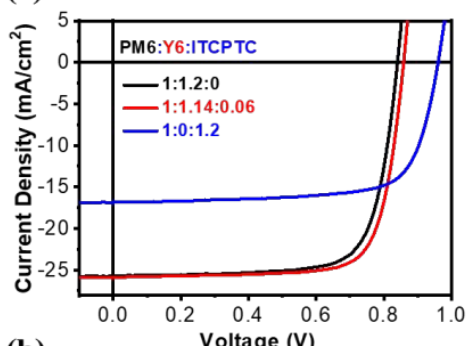

(b)

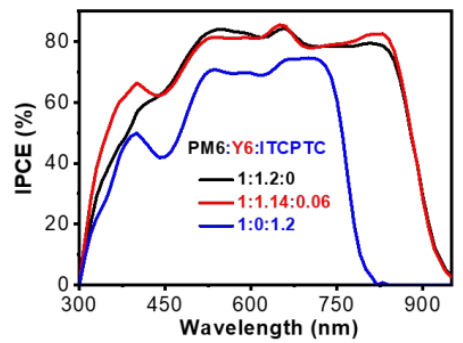

(c)

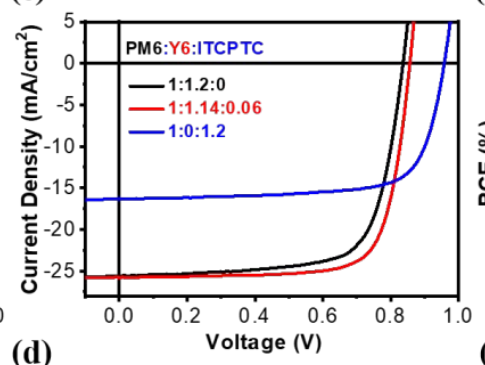

(d)

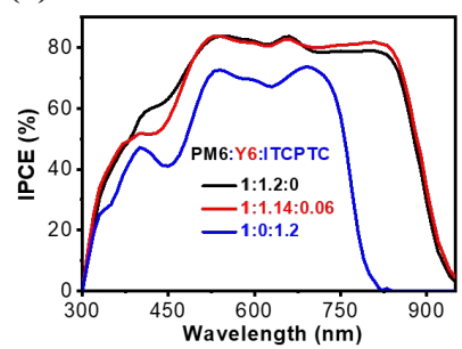

(e)

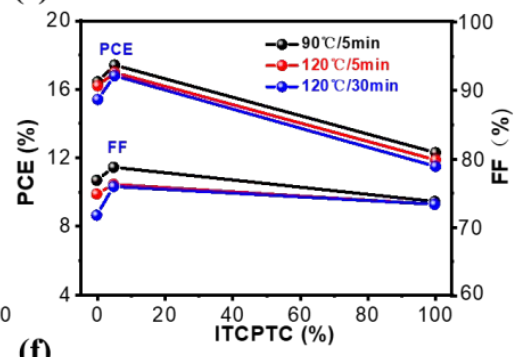

(f)

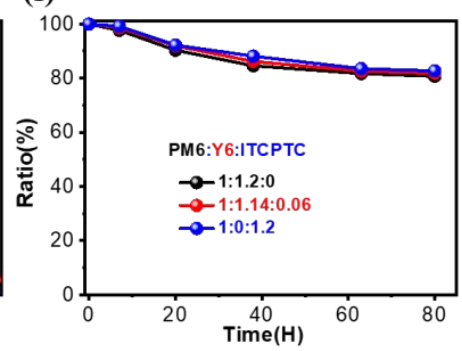

Figure S4. (a) $J-V$ characteristics and (b) IPCE spectra for devices whose active layers undergo temperature annealing at $120^{\circ} \mathrm{C}$ for $5 \mathrm{~min}$; (c) $J-V$ characteristics and (d) IPCE spectra for devices whose active layers undergo temperature annealing at $120^{\circ} \mathrm{C}$ for 30 min; (e) PCE and FF variation tendency of devices with different temperature annealing processes; (f) photostability of optimized devices based on these three combinations. 


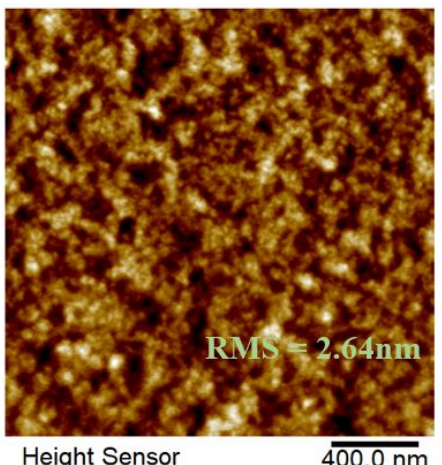

PM6:Y6

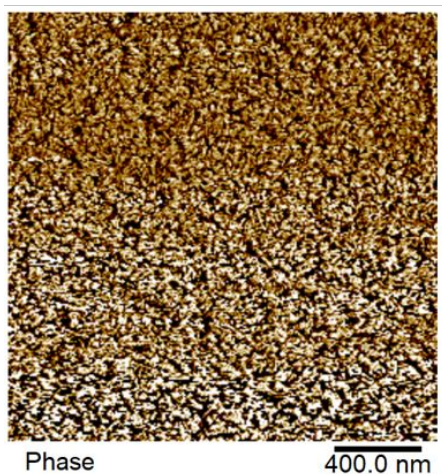

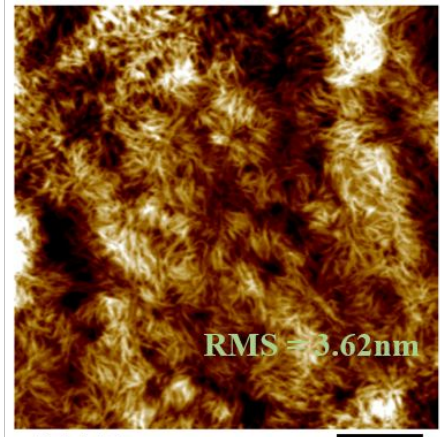

$4 \overline{00.0 \mathrm{~nm}}$

Ternary

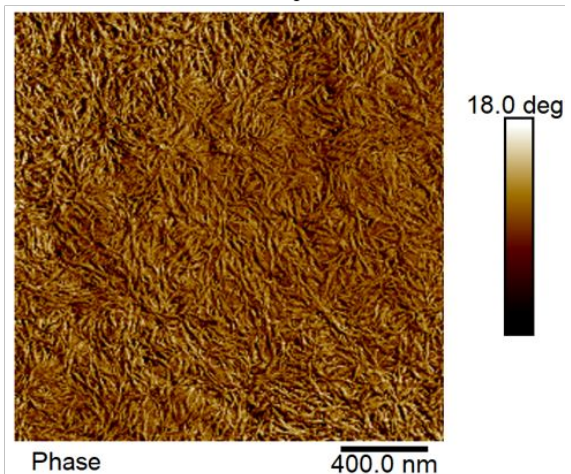

Figure S5. AFM height and phase images of PM6:Y6 and optimal ternary films after annealed at $120^{\circ} \mathrm{C}$ for $30 \mathrm{~min}$.

(a)

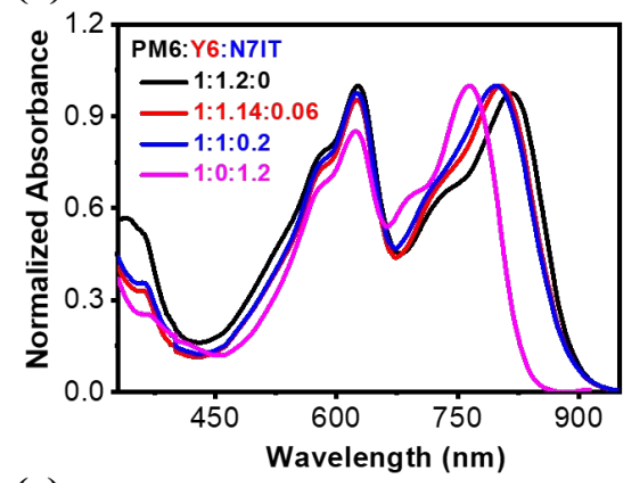

(c)

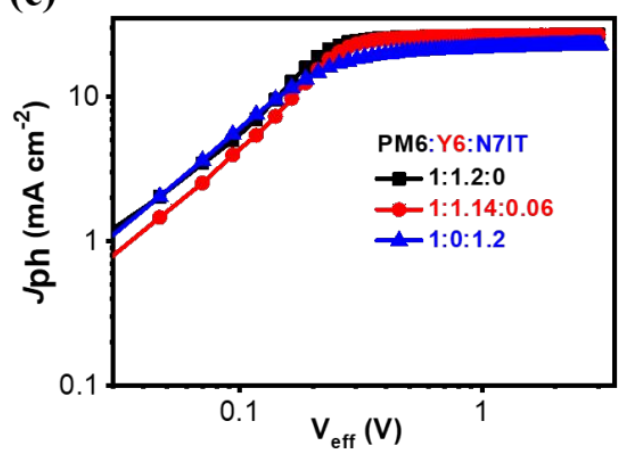

(b)

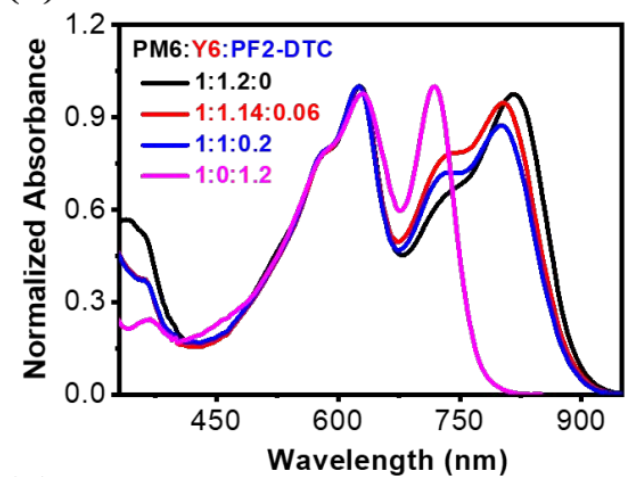

(d)

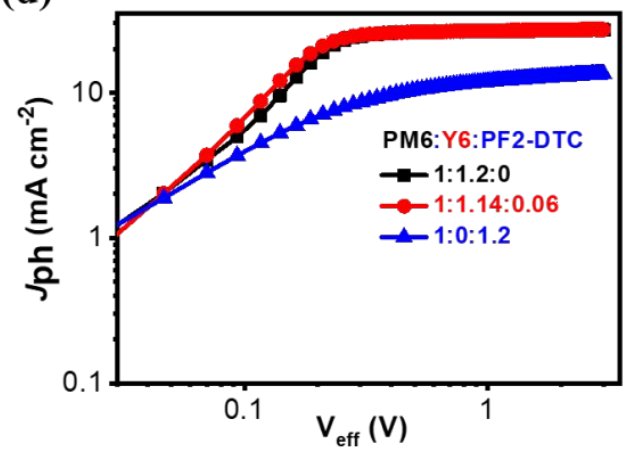

Figure S6. (a), (b) UV-vis absorption spectra, and (c), (d) $J_{\mathrm{ph}}-V_{\text {eff }}$ curves of binary and ternary films based on PM6:Y6:N7IT \& PM6:Y6:PF2-DTC of different weight ratios 


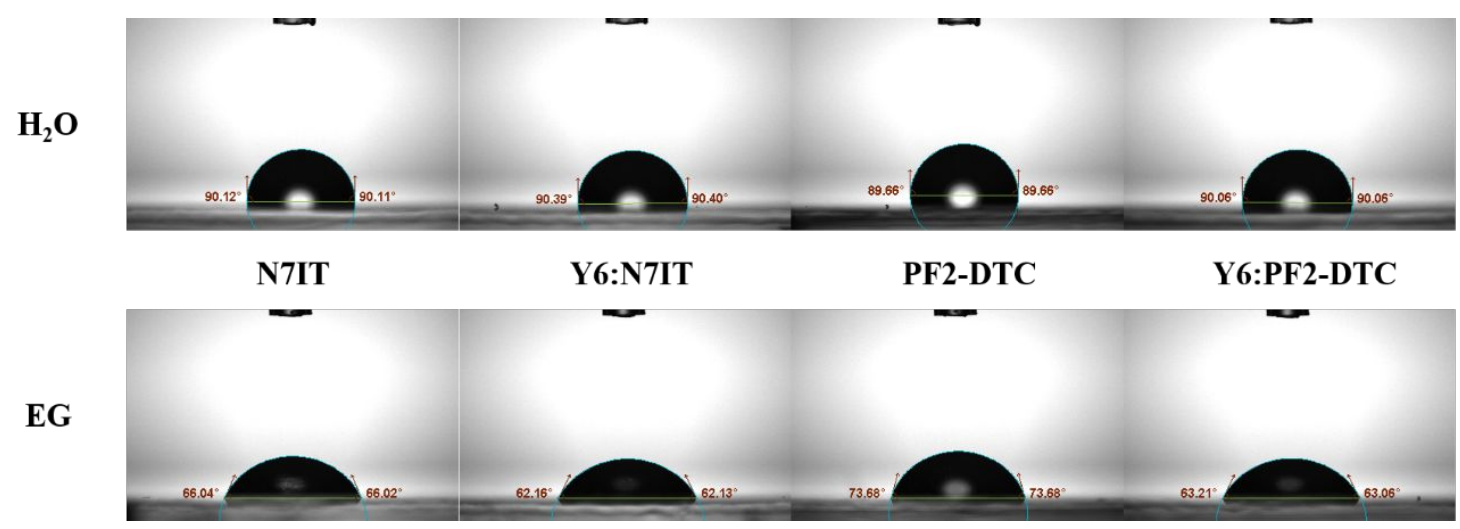

Figure S7. (a) Contact angle images of neat N7IT and PF2-DTC films, together with Y6:N7IT \& Y6:PF2-DTC $(w / w=1.14: 0.06)$ blend film on water and ethylene glycol.
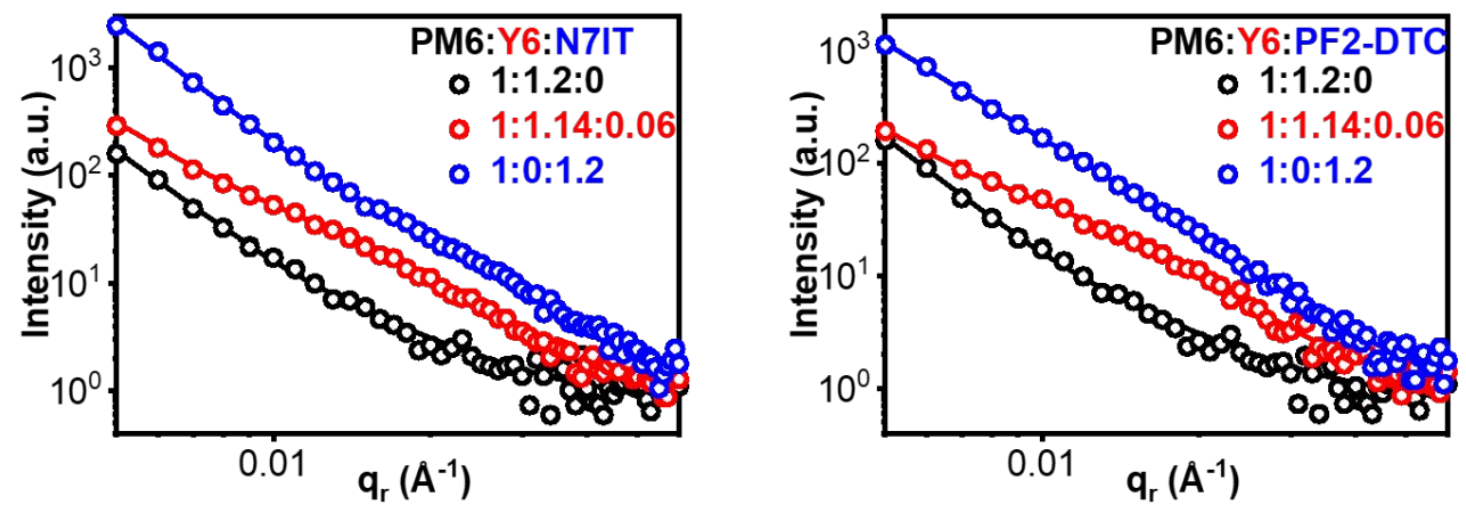

Figure S8. Cut-line profiles of GISAXS measurements of binary and ternary (N7IT and PF2-DTC, respectively) active layers. 
(a)
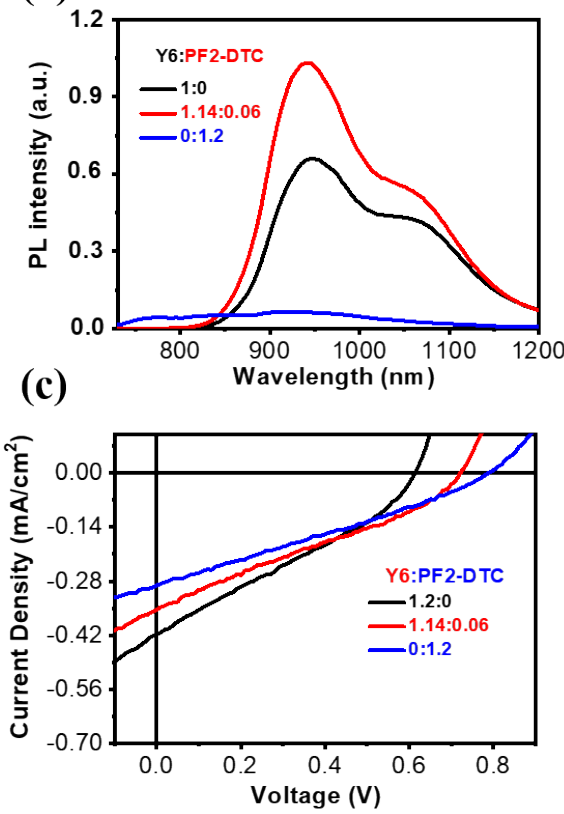

(b)
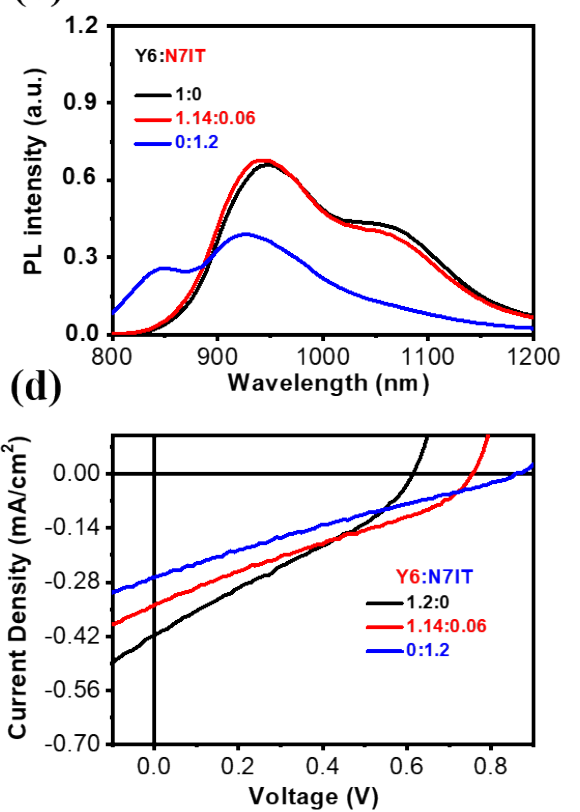

Figure S9. (a), (b) PL spectra of acceptors and (c), (d) $J-V$ characteristics of acceptoronly devices.

Table S1. Calculated results from $J_{\mathrm{ph}}-V_{\text {eff }}$ curves

\begin{tabular}{cccccc}
\hline PM6:Y6:ITCPTC & $\boldsymbol{J}_{\mathbf{s a t}^{\mathbf{c}}}$ & $\boldsymbol{J}_{\mathbf{p h}^{\mathbf{a c}}}$ & $\boldsymbol{J}_{\mathbf{p h}^{\mathbf{b c}}}$ & $\boldsymbol{J}_{\mathbf{p h}^{\mathbf{a c}}} / \boldsymbol{J}_{\mathbf{s a t}} \mathbf{c}^{\mathbf{c}}$ & $\boldsymbol{J}_{\mathbf{p h}} \mathbf{b}^{\mathbf{b c}} / \boldsymbol{J}_{\mathbf{s a t}^{\mathbf{c}}}$ \\
\hline $\mathbf{1 : 1 . 2 : 0}$ & 27.072 & 25.328 & 22.815 & $93.6 \%$ & $84.3 \%$ \\
$\mathbf{1 : 1 . 1 4 : 0 . 0 6}$ & 27.005 & 25.674 & 24.062 & $95.1 \%$ & $89.1 \%$ \\
$\mathbf{1 : 0 : 1 . 2}$ & 19.043 & 17.285 & 15.718 & $90.8 \%$ & $82.5 \%$ \\
\hline
\end{tabular}

$J_{\mathrm{ph}}$ values under (a) short-circuit and (b) maximal output condition.

(c) The unit is $\mathrm{mA} \mathrm{cm}-2$

Table S2. Blend mobilities

\begin{tabular}{lll}
\hline Blend & Hole mobility $\left(\mu_{\mathrm{h}}\right)$ & Electron mobility $\left(\mu_{\mathrm{e}}\right)$ \\
\hline PM6:Y6 & $5.84 \pm 0.9\left(10^{-4} \mathrm{~cm}^{2} \mathrm{~V}^{-1} \mathrm{~s}^{-1}\right)$ & $3.23 \pm 0.8\left(10^{-4} \mathrm{~cm}^{2} \mathrm{~V}^{-1} \mathrm{~s}^{-1}\right)$ \\
PM6:Y6:ITCPTC & $6.34 \pm 1.2\left(10^{-4} \mathrm{~cm}^{2} \mathrm{~V}^{-1} \mathrm{~s}^{-1}\right)$ & $4.15 \pm 1.1\left(10^{-4} \mathrm{~cm}^{2} \mathrm{~V}^{-1} \mathrm{~s}^{-1}\right)$ \\
PM6:ITCPTC & $5.78 \pm 0.9\left(10^{-4} \mathrm{~cm}^{2} \mathrm{~V}^{-1} \mathrm{~s}^{-1}\right)$ & $3.03 \pm 0.8\left(10^{-4} \mathrm{~cm}^{2} \mathrm{~V}^{-1} \mathrm{~s}^{-1}\right)$ \\
\hline
\end{tabular}

Results are averaged from 10 devices.

T-test results:

For $\mu_{\mathrm{h}}: \mathrm{t}_{\mathrm{PM} 6: \mathrm{Y} 6 ; \text { Ternary }}=2.357$, larger than the single side value of $\mathrm{t}_{0.025 / 18}=2.101$, significant for $97.5 \%$ confidence

For $\mu_{\mathrm{e}}: \mathrm{t}_{\mathrm{PM} 6: \mathrm{Y} 6 ; \text { Ternary }}=2.391$, larger than the single side value of $\mathrm{t}_{0.025 / 18}=2.101$, significant for $97.5 \%$ accuracy 
Table S3. Solar cell performance of binary and ternary OSCs.

\begin{tabular}{|c|c|c|c|c|c|}
\hline PM6:Y6:ITCPTC & $V_{O C}{ }^{a}(V)$ & $J_{\mathrm{SC}}\left(\mathrm{mA} \mathrm{cm} \mathrm{cm}^{-2}\right)$ & $J_{\mathrm{SC}^{\mathrm{a}}}(\mathrm{mA} \mathrm{cm}-2)$ & $F F(\%)$ & PCE (\%) \\
\hline 1:1.2:0 & 0.840 & 25.719 & 25.465 & 74.9 & 16.20 \\
\hline \multicolumn{6}{|l|}{$120^{\circ} \mathrm{C} 5 \mathrm{~min}$} \\
\hline 1:1.14:0.06 & 0.859 & 25.918 & 25.686 & 76.3 & 16.98 \\
\hline \multicolumn{6}{|l|}{$120^{\circ} \mathrm{C} 5 \mathrm{~min}$} \\
\hline 1:0:1.2 & 0.960 & 16.859 & 16.328 & 73.4 & 11.89 \\
\hline \multicolumn{6}{|l|}{$120^{\circ} \mathrm{C} 5 \mathrm{~min}$} \\
\hline 1:1.2:0 & 0.837 & 25.627 & 25.366 & 71.8 & 15.41 \\
\hline \multicolumn{6}{|l|}{$120^{\circ} \mathrm{C} 30 \mathrm{~min}$} \\
\hline 1:1.14:0.06 & 0.857 & 25.781 & 25.543 & 76.0 & 16.80 \\
\hline \multicolumn{6}{|l|}{$120^{\circ} \mathrm{C} 30 \mathrm{~min}$} \\
\hline $\begin{array}{c}\mathbf{1 : 0 : 1 . 2} \\
120^{\circ} \mathrm{C} 30 \mathrm{~min}\end{array}$ & 0.958 & 16.345 & 15.988 & 73.4 & 11.50 \\
\hline
\end{tabular}

(a) Integrated $J_{\mathrm{SC}} \mathrm{S}$ according to IPCE spectra.

Table S4. Solar cell performance of binary and ternary OSCs.

\begin{tabular}{cccccc}
\hline Active layers & $\boldsymbol{V}_{\mathbf{O C}^{\mathbf{a}}}(\mathbf{V})$ & $\boldsymbol{J}_{\mathbf{S C}^{\mathbf{a}}} \mathbf{( \mathbf { m A ~ } \mathbf { ~ c m } ^ { - 2 } )}$ & $\boldsymbol{J}_{\mathbf{S C}^{\mathbf{b}}} \mathbf{( \mathbf { m A ~ } \mathbf { ~ c m } ^ { - 2 } )}$ & $\boldsymbol{F F}^{\mathbf{a}}(\mathbf{\%})$ & $\mathbf{P C E}^{\mathbf{a}}(\mathbf{\%})$ \\
\hline PM6:Y6 & $(0.842 \pm 0.004)$ & $(25.241 \pm 0.358)$ & 25.067 & $(76.7 \pm 0.8)$ & $(16.30 \pm 0.25)$ \\
1:1.2 & 0.844 & 25.328 & & 76.9 & 16.44 \\
PM6:Y6:N7IT & $(0.854 \pm 0.005)$ & $(25.456 \pm 0.334)$ & 25.303 & $(77.2 \pm 0.8)$ & $(16.79 \pm 0.15)$ \\
1:1.14:0.06 & 0.854 & 25.538 & & 77.7 & 16.96 \\
PM6:Y6:N7IT & $(0.867 \pm 0.003)$ & $(24.356 \pm 0.329)$ & 23.917 & $(73.9 \pm 0.6)$ & $(15.62 \pm 0.14)$ \\
1:1:0.2 & 0.869 & 24.166 & & 75.1 & 15.77 \\
PM6:N7IT & $(0.942 \pm 0.005)$ & $(19.963 \pm 0.261)$ & 19.793 & $(67.9 \pm 1.8)$ & $(12.77 \pm 0.37)$ \\
1:1.2 & 0.946 & 20.077 & & 69.8 & 13.27 \\
PM6:Y6:PF2-DTC & $(0.851 \pm 0.005)$ & $(25.679 \pm 0.129)$ & 25.693 & $(77.1 \pm 0.5)$ & $(16.88 \pm 0.13)$ \\
1:1.14:0.06 & 0.854 & 25.728 & & 77.5 & 17.04 \\
PM6:Y6:PF2-DTC & $(0.865 \pm 0.003)$ & $(24.465 \pm 0.276)$ & 24.170 & $(73.6 \pm 0.5)$ & $(15.58 \pm 0.24)$ \\
1:1:0.2 & 0.866 & 24.591 & & 73.9 & 15.75 \\
PM6:PF2-DTC & $(0.935 \pm 0.003)$ & $(11.586 \pm 0.162)$ & 10.838 & $(54.6 \pm 0.6)$ & $(5.92 \pm 0.11)$ \\
1:1.2 & 0.937 & 11.534 & & 55.5 & 6.00 \\
\hline
\end{tabular}

(a) Average values based on 20 devices are put in brackets. (b) Integrated $J_{\mathrm{SC}} \mathrm{S}$ according to IPCE spectra. 
Table S5. Calculated results from $J_{\mathrm{ph}}-V_{\text {eff }}$ curves

\begin{tabular}{|c|c|c|c|c|c|}
\hline Active layers & $J_{\text {sat }}{ }^{\mathrm{c}}$ & $J_{\mathrm{ph}}{ }^{\text {ac }}$ & $J_{\mathrm{ph}}{ }^{\mathrm{bc}}$ & $J_{\mathrm{ph}}^{\mathrm{ac}} / J_{\mathrm{sat}}^{\mathrm{c}}$ & $J_{\text {ph }}{\text { bc } / J_{\text {sat }}}^{\mathrm{c}}$ \\
\hline \multicolumn{6}{|l|}{ PM6:Y6:N7IT } \\
\hline 1:1.2:0 & 27.072 & 25.328 & 22.815 & $93.6 \%$ & $84.3 \%$ \\
\hline 1:1.14:0.06 & 26.935 & 25.538 & 23.207 & $94.8 \%$ & $86.2 \%$ \\
\hline $1: 0: 1.2$ & 22.824 & 20.077 & 17.982 & $88.0 \%$ & $78.8 \%$ \\
\hline \multicolumn{6}{|l|}{ PM6:Y6:PF2-DTC } \\
\hline 1:1.2:0 & 27.072 & 25.328 & 22.815 & $93.6 \%$ & $84.3 \%$ \\
\hline 1:1.14:0.06 & 27.235 & 25.728 & 23.337 & $94.5 \%$ & $85.6 \%$ \\
\hline 1:0:1.2 & 13.443 & 11.543 & 7.861 & $85.8 \%$ & $58.5 \%$ \\
\hline
\end{tabular}

$J_{\text {ph }}$ values under (a) short-circuit and (b) maximal output condition.

(c) The unit is $\mathrm{mA} \mathrm{cm} \mathrm{cm}^{-2}$

Table S6. Contact angles, surface tensions $\gamma$, and interaction parameters $\chi$.

\begin{tabular}{ccccc}
\hline Surface & $\boldsymbol{\theta}_{\text {water }}\left({ }^{\circ}\right)$ & $\boldsymbol{\theta}_{\text {EG }}\left(^{\circ}\right)$ & $\gamma\left(\mathbf{m N ~ m}^{-1}\right)^{\mathrm{a}}$ & $\chi^{\mathbf{D}-\mathrm{Ab}}$ \\
\hline PM6 & 107.17 & 75.57 & 32.008 & \\
Y6 & 90.41 & 59.17 & 33.495 & 0.017 \\
N7IT & 90.12 & 66.03 & 25.270 & 0.398 \\
Y6:N7IT & 90.39 & 60.14 & 29.692 & 0.043 \\
PF2-DTC & 89.66 & 73.68 & 19.740 & 1.475 \\
Y6:PF2-DTC & 90.06 & 63.13 & 28.245 & 0.118 \\
\hline
\end{tabular}

(a) The Flory-Huggins interaction parameter based on the surface tension data formula,

(b) The Flory-Huggins interaction parameters between the donor (D) and the acceptor (A) calculated using $\chi=(\sqrt{\gamma \mathrm{D}}-\sqrt{\gamma \mathrm{A}})^{2}$

\section{Reference}

[1] Z. Wu, C. Sun, S. Dong, X.-F. Jiang, S. Wu, H. Wu, H.-L. Yip, F. Huang and Y. Cao, J Am Chem Soc., 2016, 138, 2004-2013.

[2] Fowkes, F. M., ATTRACTIVE FORCES AT INTERFACES. Industrial \& Engineering Chemistry 1964, 56 (12), 40-52. 